\title{
Chromophores from hexeneuronic acids: identification of HexA-derived chromophores
}

\author{
Thomas Rosenau • Antje Potthast • Nele Sophie Zwirchmayr • Hubert Hettegger • \\ Felix Plasser - Takashi Hosoya - Markus Bacher - Karin Krainz • \\ Thomas Dietz
}

Received: 9 April 2017 / Accepted: 1 July 2017/Published online: 13 July 2017

(C) The Author(s) 2017. This article is an open access publication

\begin{abstract}
Hexeneuronic acids (HexA) have long been known as triggers for discoloration processes in glucuronoxylan-containing cellulosic pulps. They are formed under the conditions of pulping from 4-Omethylglucuronic acid residues, and are removed in an "A stage" along the bleaching sequences, which mainly comprises acidic washing treatments. The chemical structures of HexA-derived chromophoric compounds 4-8, which make up $90 \%$ of the HexAderived chromophores, are reported here for the first time. The compounds are ladder-type, mixed quinoidaromatic oligomers of the bis(furano)-[1,4]benzoquinone and bis(benzofurano)-[1,4]benzoquinone type. The same chromophoric compounds are generated independently of the starting material, which can
\end{abstract}

Chromophores in cellulosic materials. Part XVI.

T. Rosenau ( $\square)$. A. Potthast · N. S. Zwirchmayr .

H. Hettegger - T. Hosoya · M. Bacher

Division of Chemistry of Renewable Resources,

Department of Chemistry, BOKU University Vienna,

Muthgasse 18, 1190 Vienna, Austria

e-mail: thomas.rosenau@boku.ac.at

F. Plasser

Faculty of Chemistry, Institute for Theoretical Chemistry, University of Vienna, Währingerstr. 17, 1090 Vienna,

Austria

K. Krainz

Air Liquid GmbH, Sendnergasse 30, 2320 Schwechat, Austria be either a) HexA in pulp, b) the HexA model compound methyl $1-{ }^{13} \mathrm{C}-4$-deoxy- $\beta$-L-threo-hex-4enopyranosiduronic acid (1) or c) a mixture of the primary degradation intermediates of $\mathbf{1}$, namely 5-formyl-furancarboxylic acid (2) and 2-furancarboxylic acid (3). Isotopic labeling $\left({ }^{13} \mathrm{C}\right)$ in combination with NMR spectroscopy and mass spectrometry served for structure elucidation, and final confirmation was provided by $\mathrm{X}$-ray structure analysis. ${ }^{13} \mathrm{C}$-Isotopic labeling was also used to establish the formation mechanisms, showing all the compounds to be composed of condensed, but otherwise largely intact, 2-carbonylfuran and 2-carbonylfuran-5-carboxylic acid moieties. These results disprove the frequent assumption that HexA-derived or furfural-derived chromophores are linear furanoid polymers, and might have a direct bearing on structure elucidation studies

T. Dietz

Evonik-Degussa, Rodenbacher Chaussee 4,

63457 Hanau-Wolfgang, Germany

T. Rosenau

Johan Gadolin Process Chemistry Centre, Åbo Akademi

University, Porthansgatan 3, 20500 Turku, Finland 
of "humins", which are formed as dark-colored byproducts in depolymerization of pentosans and hexosans in different biorefinery scenarios.

Keywords Cellulose · Pulp · Chromophores · Hexeneuronic acids · Bleaching - A-stage · Furan · Furancarboxylic acid $\cdot$ Ladder-type oligomers

\section{Introduction}

The pulp and paper industry is a major economic factor in many countries. Today, with biorefinery topics becoming more and more prominent, paper mills are in transition from classical pulp producers to more diverse biorefineries. But still, the major product of these industries is cellulose, with pulping and bleaching being the two main operations in its manufacture. A major part of chemical, energy and technology costs is devoted to removing chromophores from pulp to reach target brightness. The fewer chromophores are left in pulp, the more difficult it gets to remove the remaining ones. For glucuronoxylan-containing pulps, the role of hexeneuronic acids (HexA) has been recognized as critical with regard to color formation ("brightness reversion"). The origin of this moiety in pulp has been briefly repeated in the previous part of this series (Rosenau et al. 2017).

Studies by Clayton dating back to 1963 investigated the formation of 4-deoxy- $\beta$-L-threo-hex-4- enopyranosiduronic acid (HexA) moieties from hemicelluloses during alkaline treatment of wood at temperatures up to $170{ }^{\circ} \mathrm{C}$. From $4-O$-methyl glucopyranosiduronic acid, HexA formation was suggested to occur through $\beta$-elimination of methanol, leaving a double bond that is always placed regioselectively towards $\mathrm{C}-5$, thus resulting in a conjugated $\alpha, \beta$-unsaturated acid structure (see Scheme 1) (Clayton 1963). Further studies were able to confirm these early findings (Johansson and Samuelson 1977), establishing definite knowledge of the structure of HexA, when enzymatic hydrolysis was applied to kraft pulps by Teleman et al. (1995). The role of HexA in decreasing brightness has been thoroughly investigated, as were the bleaching conditions that permit its minimization (Teleman et al. 1995; Sjostrom 2006). Acidic pulping conditions, e.g. at $130{ }^{\circ} \mathrm{C}$ for $2-3 \mathrm{~h}$ with $0.05 \mathrm{M} \mathrm{H}_{2} \mathrm{SO}_{4}$ (Johansson and Samuelson 1977), or at 80 to $140{ }^{\circ} \mathrm{C}$ and $\mathrm{pH} 3.0-3.5$ for $2-5 \mathrm{~h}$ (Chakar et al. 2000; Clavijo et al. 2012), allow the removal of possible HexA side groups, but also result in the occurrence of furanoid primary degradation products, e.g. 2-furancarboxylic acid and 5-formyl-2-furancarboxylic acid that lead to the formation of hitherto unidentified chromophoric compounds (Clavijo et al. 2012; Teleman et al. 1996). Analyses have focused mainly on determining the amount of uronic acids; and the resulting HexA moieties, present in the pulp and methods to inhibit the brightness reversion (Granstrom and Eriksson 2001; Forsskåhl et al. 2000; Li and Gellerstedt 1997), but the primary degradation
Scheme 1 Top: schematic formation of HexA from glucuronoxylan during pulping; bottom: structure of the HexA model compound methyl 4-deoxy- $\beta$-L-threohex-4-enopyranosiduronic acid (1) and its primary degradation products 5-formylfuran-2-carboxylic acid (2) and furan-2carboxylic acid (3)
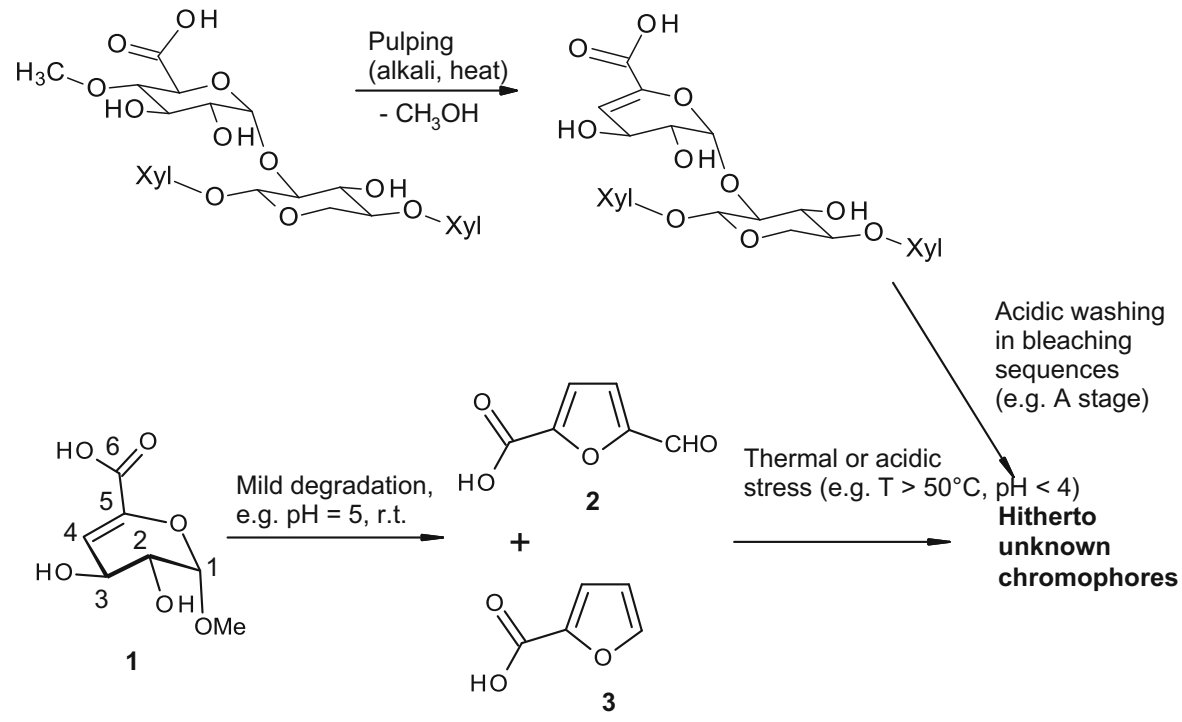
products and the secondary chromophores formed from them had received less attention. Some studies suggested the formation of 2,3-dihydroxy-2-cyclopenten-1-one (reductic acid) from HexA and its involvement in chromophore formation (Sevastyanova et al. 2006a, b).

By applying the well-established technique of CRI (chromophore release and identification method, see also the previous parts of this series) (Rosenau et al. 2004, 2005, 2007; Korntner et al. 2015), identification of the chromophores formed from HexA or from its furanoid primary degradation products was possible, and their structures-all of them being of the bis(furano)-[1,4]benzoquinone or bis(benzofurano)$[1,4]$ benzoquinone type-are hereby reported.

\section{Materials and methods}

\section{General}

Commercial chemicals were of the highest grade available and were used without further purification. ${ }^{1}$ Reagent-grade solvents were used for all extractions and workup procedures. Distilled water was used for all aqueous extractions and for all aqueous solutions. $n$-Hexane, diethyl ether, ethyl acetate, and petroleum ether for chromatography were distilled before use. All reactions involving non-aqueous conditions were conducted in oven-dried $\left(140{ }^{\circ} \mathrm{C}\right.$, overnight $)$ or flamedried glassware under an inert argon or nitrogen atmosphere. TLC was performed using Merck silica gel $60 \mathrm{~F}_{254}$ pre-coated plates. Flash column chromatography was performed using Baker silica gel (40 $\mu \mathrm{m}$ particle size). All products were purified to homogeneity by TLC/GCMS analysis. The use of brine refers to saturated aqueous $\mathrm{NaCl}$ solution. All given yields refer to isolated, pure products.

${ }^{1} \mathrm{H}$ NMR spectra were recorded at $400.13 \mathrm{MHz}$ for ${ }^{1} \mathrm{H}$ and at $100.62 \mathrm{MHz}$ for ${ }^{13} \mathrm{C} \mathrm{NMR}$ in $\mathrm{CDCl}_{3}$ at room temperature, if not stated otherwise. Chemical shifts, relative to tetramethylsilane (TMS) as the internal standard, are given in $\delta \mathrm{ppm}$ values, and coupling

\footnotetext{
1 Note: this part on general analysis is equal to the corresponding description part in the preceeding part of this series as the work is closely related-no unlawful duplication is intended.
}

constants are given in $\mathrm{Hz} .{ }^{13} \mathrm{C}$ peaks were assigned by means of high-resolved $1 \mathrm{D}-{ }^{13} \mathrm{C}$, APT, HSQC and HMBC spectra.

GCMS analyses were carried out on an Agilent $6890 \mathrm{~N} / 5975 \mathrm{~B}$ instrument in the ESI $(70 \mathrm{eV})$ ionization mode. Melting points, determined on a Koflertype micro hot stage with Reichert-Biovar microscope, are uncorrected. Elemental analyses were performed at the Microanalytical Laboratory at the University of Vienna. All compounds showed satisfactory microanalytical data within the limits of $0.24 \%$.

\section{Starting materials}

Two pulp samples were obtained from commercial suppliers, chosen according to known high HexA contents to enable isolation of as "large" chromophore amounts as possible. Relevant pulp data including the methods of determination are summarized in Table 1. The initial brightness of the dry samples (89\%ISO for pulp I and 90\%ISO for pulp II) dropped to 74 and $79 \%$ ISO, respectively, after dry aging and 68 and $72 \%$ ISO, respectively, after aging in humid medium. Accelerated aging was performed under daylight in $4 \mathrm{~h}$ intervals at $105{ }^{\circ} \mathrm{C}$ according to TAPPI UM 200 and $1 \mathrm{~h}$ intervals at $100{ }^{\circ} \mathrm{C}$ and $100 \%$ humidity according to Paptac E.4P. Brightness was measured as UV/Vis reflection at $457 \mathrm{~nm}$ according to ISO 3688/2470.

\section{GPC and CCOA method}

The gel permeation chromatography of celluloses with concomitant Mw-related profiling of carbonyl groups (Potthast et al. 2005) ("CCOA method") (Röhrling et al. 2002a, b; Potthast et al. 2003) and carboxyl groups ("FDAM method") (Bohrn et al. 2005, 2006) was based on multiple detection by refractive index (RI), multi-angle laser light scattering (MALLS) and fluorescence, using the setup of instruments and protocol of the selective fluorescence labeling ("CCOA method") as previously reported. The method was applied to characterization of the different pulp samples. Especially the carboxyl-selective FDAM technique is useful in the present case to reflect changes in HexA content through the inherent carboxylic moieties. 
Table 1 Analytical data of the two cellulosic pulps used

\begin{tabular}{|c|c|c|}
\hline & Pulp 1 & Pulp 2 \\
\hline Wood source & Eucalyptus & Eucalyptus \\
\hline Pulping technology & Kraft & Kraft \\
\hline Bleaching sequence & $\mathrm{D}_{0}-\mathrm{EOP}-\mathrm{D}(\mathrm{ECF})$ & O-P-P (TCF) \\
\hline Pulp producer & Suzano, Brazil & Ence, Spain \\
\hline Molar mass $^{\mathrm{a}}(\mathrm{g} / \mathrm{mol})$ & 418.000 & 622.000 \\
\hline Carbonyl group content ${ }^{\mathrm{a}}(\mu \mathrm{mol} / \mathrm{g})$ & 8 & 20 \\
\hline Carboxyl group content ${ }^{\mathrm{b}}(\mu \mathrm{mol} / \mathrm{g})$ & 42 & 102 \\
\hline HexA content ${ }^{\mathrm{c}}(\mu \mathrm{mol} / \mathrm{g})$ & 8.1 & 22.3 \\
\hline Brightness $^{\mathrm{d}}$ (\% ISO) & 89.1 & 90.1 \\
\hline Carbohydrate composition $^{\mathrm{e}}(\% 0)$ & Gal $0.2 \%$, Glu $91.8 \%$, Xyl $6.5 \%$ & Gal $0.3 \%$, Glu $79.3 \%$, Xyl $19.1 \%$ \\
\hline Kappa number ${ }^{\mathrm{f}}$ & $<1$ & 5.99 \\
\hline \multicolumn{3}{|c|}{$\begin{array}{l}\text { Simultaneous determination of molar mass distribution and carbonyl group profiles according to the CCOA method (Röhrling et al. } \\
\text { 2002a, b; Potthast et al. 2003) }\end{array}$} \\
\hline \multicolumn{3}{|c|}{ b Determination of the carboxyl group content according to the FDAM method (Bohrn et al. 2005, 2006) } \\
\hline \multicolumn{3}{|c|}{ c Determined according to T 282 pm-07 "Hexeneuronic acid content of chemical pulp" (pm-07 2009) } \\
\hline \multicolumn{3}{|c|}{$\begin{array}{l}\text { d Determined according to "ISO 2470-1:2009 Paper, board and pulps-measurement of diffuse blue reflectance factor-part 1: } \\
\text { indoor daylight conditions (ISO brightness)" (ISO 2470) }\end{array}$} \\
\hline \multicolumn{3}{|l|}{ e Based on total hydrolysis } \\
\hline
\end{tabular}

Isolation of HexA-derived chromophores

from pulp according to the CRI method

The CRI method was used as previously published (Rosenau et al. 2004, 2007). In summary, the pulp was suspended in a highly pure (bidistilled) solvent in the presence of $\mathrm{BF}_{3}$ acetate complex/sodium sulfite as catalysts. CRI was applied to $2.8 \mathrm{~kg}$ of the pulp samples to obtain chromophores in sufficiently "large" amounts. This treatment releases all electron-rich aromatic and/or quinoid compounds, which are subsequently chromatographically separated under careful exclusion of oxygen and in the presence of tocopherol antioxidants in the eluent.

The amount of the individual components was between 0.3 and $1.2 \mathrm{mg}$, which normally would have been sufficient to derive the structure of the compounds by a combination of NMR, IR and MS. Due to the highly symmetric structure of the compounds, the spectra (especially in ${ }^{1} \mathrm{H}$ and ${ }^{13} \mathrm{C}$ NMR) were not highly characteristic and revealing. Fortunately, the high tendency of the compounds to crystallize could be used to establish their identity by single crystal $\mathrm{X}$-ray structure as compounds $\mathbf{4 - 8}$, which was then correlated with the results of the spectroscopic techniques.

Comparison of the analytical data showed the compounds to be identical to those obtained from HexA model compound $\mathbf{1}$ and a mixture of its primary degradation products 5-formylfuran-2-carboxylic acid (2) and furan-2-carboxylic acid (3), see below.

Isolation of HexA-derived chromophores from model compound $\mathbf{1}$

Methyl 4-deoxy- $\beta$-L-threo-hex-4-enopyranosiduronic acid (1, $190 \mathrm{mg}, 1 \mathrm{mmol})$, or its isotopomers 1a-1f, were dissolved in $10 \mathrm{ml}$ of sulfuric acid ( $\mathrm{pH} 0$ ) which had been deaerated by flushing with argon for $5 \mathrm{~min}$. The mixture was stirred under argon and under exclusion of light (vessel wrapped in aluminum foil) at $50{ }^{\circ} \mathrm{C}$. The solution turned yellow immediately and the color changed gradually into dark brown within $10 \mathrm{~min}$. After about $30 \mathrm{~min}$ a brownish-black precipitate formed while at the same time the liquid phase turned nearly colorless again. TLC analysis (MeOH/ $\left.\mathrm{CH}_{2} \mathrm{Cl}_{2} / \mathrm{H}_{2} \mathrm{O}, \quad \mathrm{v} / \mathrm{v} / \mathrm{v}=5: 10: 1\right)$ of the precipitate showed the presence of 5 compounds which did not 
change over prolonged stirring. After $2 \mathrm{~h}$ the stirring was discontinued and the solution cooled to r.t. TLC control showed the liquid phase to be free from organic solutes, indicating complete conversion of initially soluble organics into the insoluble black precipitate. The precipitate was washed with distilled water until the washings were neutral, and subsequently with $5 \mathrm{ml}$ of diethylether to remove adhering water. The precipitate was dissolved in $2 \mathrm{ml}$ of $\mathrm{N}, \mathrm{N}$ dimethylformamide, diluted with $5 \mathrm{ml}$ of dry toluene (HPLC grade), transferred to a chromatographic column and chromatographed on silica gel using toluene/ethanol $(\mathrm{v} / \mathrm{v}=95: 5)$ as the eluent, with the ethanol component in the binary solvent system significantly improving the separation of the carboxyl-containing compounds as compared to pure toluene. Five well-separable compounds were eluted, corresponding to $92 \%$ of the starting mass.

A mixture of trace compounds, less colored than the main products and corresponding to $3 \%$ of the starting mass, was eluted with toluene/ethanol $(\mathrm{v} / \mathrm{v}=85: 15)$ at $45{ }^{\circ} \mathrm{C}$. This mixture proved to be inseparable; it formed a brownish film upon drying that proved to be completely insoluble in all solvents tried, also under prolonged stirring and warming. Due to its mass-wise insignificance, this side product mixture was not further studied and discarded.

The five main compounds eluted were identical to compounds 4-8 as identified above. Elution order under the condition applied was $4-6-8-7-5$.

Isolation of HexA-derived chromophores

from the primary degradation products of HexA, 5-formylfuran-2-carboxylic acid (2) and furan-2carboxylic acid (3)

A mixture of 5-formylfuran-2-carboxylic acid (2) and furan-2-carboxylic acid (3) was prepared by careful, mildly acidic treatment ( $\mathrm{pH} 4.5$ in aqueous phosphate buffer, r.t.) of methyl 4-deoxy- $\beta$-L-threo-hex-4enopyranosiduronic acid (1, $190 \mathrm{mg}, 1 \mathrm{mmol})$, according to the procedure given in the preceding part of this series. The reaction mixture was adjusted to $\mathrm{pH}$ 0 with $1 N$ sulfuric acid and further treated as given above for the direct degradation of $\mathbf{1}$.

Alternatively, a 2:1 mixture (mol/mol) of 5-formylfuran-2-carboxylic acid (2) and furan-2-carboxylic acid (3) or the respective isotopomers (2a-f and $\mathbf{3 b}-\mathbf{f})$ was dissolved in sulfuric acid $(\mathrm{pH} 0)$ and was further treated as above. A changed ratio between $\mathbf{2}$ and $\mathbf{3}$ changed the ratio among the five chromatographically isolated compounds, but did not afford any other different compounds than those five identified above.

Analytical data of the HexA-derived chromophores (4-8)

The following section lists the analytical data of the five chromophores isolated from hexeneuronic acid moieties, both in pulp and model compounds. The $\mathrm{X}$-ray diffraction data have been deposited at the Cambridge Structural Database (CSD) and can be obtained for each of the five compounds free of charge. $^{2}$

Furo[2,3- $f$ ]benzofuran-4,8-dione (4). $\mathrm{M}=188.14$ g mol ${ }^{-1}$. Calcd. for $\mathrm{C}_{10} \mathrm{H}_{4} \mathrm{O}_{4}$ : C 63.84, $\mathrm{H} \mathrm{2.14}$; found C 63.94, H 2.19. TLC: toluene/ethanol $=90: 10$, $\mathrm{R}_{f}=0.42$. m.p. $=201-204{ }^{\circ} \mathrm{C}$ (literature: m.p. $>185^{\circ} \mathrm{C}$ ) (Takahashi and Kobayashi 2000). UV/Vis $\left(\mathrm{CHCl}_{3}\right): \lambda_{\max }[\mathrm{nm}](\log \varepsilon) 315$ (3.8), 255 (4.0), 245 (4.0), 230 (4.3). MS $m / z$ 188.2 (M $\left.{ }^{+}\right)$. ${ }^{1} \mathrm{H}$ NMR: $\delta 7.73$ $(\mathrm{d}, J=1.8 \mathrm{~Hz}, 2 \mathrm{H}, \mathrm{H}-3 / \mathrm{H}-7), 6.94(\mathrm{~d}, J=1.8 \mathrm{~Hz}$, 2H, H-2/H-6). ${ }^{13} \mathrm{C}$ NMR: $\delta 170.9$ (C-4/C-8), 152.0 (C4a/C-8a), 148.2 (C-2/C-6), 128.4 (C-3a/C-7a), 108.3 (C-3/C-7). The NMR data agree with those given in the literature within limits of less than $0.03 \mathrm{ppm}$ for ${ }^{1} \mathrm{H}$ and less than $0.4 \mathrm{ppm}$ for ${ }^{13} \mathrm{C}$ (Takahashi and Kobayashi 2000).

4,8-Dioxofuro[2,3-f] benzofuran-2,6-dicarboxylic acid (5). $\mathrm{M}=276.16 \mathrm{~g} \mathrm{~mol}^{-1}$. Calcd. for $\mathrm{C}_{12} \mathrm{H}_{4} \mathrm{O}_{8}: \mathrm{C}$ 52.19, H 1.46; found C 52.25, H 1.42. TLC: toluene/ ethanol $=90: 10, \quad \mathrm{R}_{f}=0.94 ; \quad$ toluene/ethanol $=75: 25, \mathrm{R}_{f}=0.71$. m.p. $>230{ }^{\circ} \mathrm{C}$ (decomp.). MS $m / z 276.3\left(\mathrm{M}^{+}\right) .{ }^{1} \mathrm{H}$ NMR: $\delta 7.24$ (s, 2H, H-3/H-7), 6.30 (s, br, 2H, COOH). ${ }^{13} \mathrm{C}$ NMR: $\delta 170.9$ (C-4/C-8), 163.9 (2-COOH/6-COOH), 151.9 (C-2/C-6), 151.4 (C-4a/C-8a), 129.1 (C-3a/C-7a), 114.0 (C-3/C-7).

5-Hydroxy-4,10-dioxo-benzofuro[5,6- $b$ ] benzofuran-8-carboxylic acid (6). $\mathrm{M}=298.20 \mathrm{~g} \mathrm{~mol}^{-1}$. Calcd. for $\mathrm{C}_{15} \mathrm{H}_{6} \mathrm{O}_{7}$ : C 60.42, H 2.03; found C 60.31, $\mathrm{H}$ 2.18. TLC: toluene/ethanol $=90: 10, \mathrm{R}_{f}=0.60$. m.p. $>255{ }^{\circ} \mathrm{C}$ (decomp.). MS $\mathrm{m} / z 298.4\left(\mathrm{M}^{+}\right) .{ }^{1} \mathrm{H}$ NMR: $\delta 10.3(\mathrm{~s}, 1 \mathrm{H}, \mathrm{COOH}), 7.73(\mathrm{~d}, J=1.8 \mathrm{~Hz}, 1 \mathrm{H}$, $\mathrm{H}-1), 7.64$ (d, $J=8.4 \mathrm{~Hz}, 1 \mathrm{H}, \mathrm{H}-7), 6.92$ (d, $J=1.8 \mathrm{~Hz}, 1-\mathrm{H}, \mathrm{H}-2), 6.55$ (d, $J=8.4 \mathrm{~Hz}, 1 \mathrm{H}$,

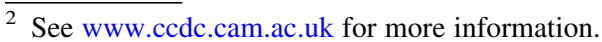


H-6). ${ }^{13} \mathrm{C}$ NMR: $\delta 175.0$ (C-4), 172.2 (C-10), 167.3 $(\mathrm{COOH}), 157.0(\mathrm{C}-5), 151.4$ (C-2), 150.9 (C-3a), 149.4 (C-8a), 149.2 (C-9a), 131.3 (C-7), 130.9 (C10a), 124.8 (C-4a), 116.8 (C-4b), 114.6 (C-8), 114.3 (C-6), 108.9 (C-1).

1,7-Dihydroxy-6,12-dioxo-6,12-dihydrobenzo[1,2- $b: 4,5-b^{\prime}$ ']bisbenzofuran-4,10-dicar-boxylic acid (7). $\mathrm{M}=408.27 \mathrm{~g} \mathrm{~mol}^{-1}$. Calcd. for $\mathrm{C}_{20} \mathrm{H}_{8} \mathrm{O}_{10}$ : C 58.84, H 1.98; found C 58.72, H 2.08. TLC: toluene/ ethanol $=90: 10, \quad \mathrm{R}_{f}=0.75$. m.p. $>280{ }^{\circ} \mathrm{C} \quad($ decomp.). MS $m / z 408.1\left(\mathrm{M}^{+}\right) .{ }^{1} \mathrm{H}$ NMR: $\delta 7.75(\mathrm{~d}$, $J=8.6 \mathrm{~Hz}, 2 \mathrm{H}, \mathrm{H}-3 / \mathrm{H}-9), 6.95$ (d, $J=8.6 \mathrm{~Hz}, 2 \mathrm{H}$, $\mathrm{H}-2, \mathrm{H}-8) .{ }^{13} \mathrm{C}$ NMR: $\delta 175.0$ (C-6/C-12), 166.3 $(\mathrm{COOH} / \mathrm{COOH}), 156.7$ (C-1/C-7), 150.2 (C-4a/C10a), 148.1 (C-5a/C-11a), 133.9 (C-3/C-9), 127.8 (C-6a/C-12a), 115.0 (C-6b/C-12b), 114.8 (C-4/C-10), 114.3 (C-2/C-8).

Benzo[1,2- $\left.b: 4,5-b^{\prime}\right][3 a, 14 a: 7 a, 10 a] d i f u r a n-b i s b e n-$ zofuran-4,6,7,11,13,14-hexaone (8). M $=428.26 \mathrm{~g}$ $\mathrm{mol}^{-1}$. Calcd. for $\mathrm{C}_{22} \mathrm{H}_{4} \mathrm{O}_{10}$ : C 61.70, $\mathrm{H} \mathrm{0.94;} \mathrm{found}$ C 61.93, H 0.94. TLC: toluene/ethanol = 90:10, $\mathrm{R}_{f}=0.68$. m.p. $>230{ }^{\circ} \mathrm{C}$ (decomp.). MS $m / z 428.5$ $\left(\mathrm{M}^{+}\right) .{ }^{1} \mathrm{H}$ NMR: $\delta 7.75(\mathrm{~d}, J=1.8 \mathrm{~Hz}, 2 \mathrm{H}, \mathrm{H}-3 / \mathrm{H}-$ 10), 6.95 (d, $J=1.8 \mathrm{~Hz}, 2 \mathrm{H}, \mathrm{H}-2 / \mathrm{H}-9) .{ }^{13} \mathrm{C}$ NMR: $\delta$ 172.6 (C-4/C-11), 172.0 (C-6/C-13), 170.2 (C-7/C14), 154.9 (C-4a/C-11a), 154.4 (C-5a/C-12a), 153.4 (C-7a/C-14a), 148.2 (C-2/C-9), 126.8 (C-3a/C-10a), 123.2 (C-6b/C-13b), 123.0 (C-6a/C-13a), 108.3 (C-3/ C-10).

\section{Results and discussion}

Structure of the HexA-derived chromophores

The CRI procedure was applied to Eucalyptus pulp with high glucuronoxylan content, which had been aged before at elevated temperature in dry environment. This protocol, applied to $2.8 \mathrm{~kg}$ of pulp, afforded a mixture of chromophores from which five different, well-defined compounds were isolated (structures shown in Scheme 2). All of the five compounds are strongly colored and thus potent chromophores. The five compounds account for $92 \%$ of the isolated chromophoric matter, the residual $8 \%$ consisting of an intractable black remainder. When the pulp was pre-aged before chromophore isolation in a humid environment, but under otherwise identical conditions, the same number of chromophores was
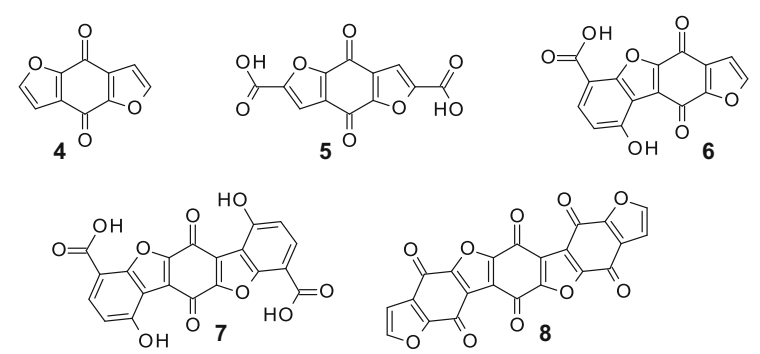

Scheme 2 Chemical structures of the five HexA-derived chromophores isolated

found, and their sum amounted to $86 \%$ of the total chromophore mixture.

In a next set of experiments, the HexA model compound methyl 4-deoxy- $\beta$-L-threo-hex-4-enopyranosiduronic acid $(\mathbf{1})^{3}$ was used as the starting material and was subject to thermal and acidic stress-under conditions similar to industrial acidic washing stages. The black precipitate obtained was separated by column chromatography and provided a mixture of five well-defined compounds that, most notably, were identical to those obtained from the pulp. In the model compound case, the combined mass of the five chromophoric compounds amounted almost to the whole mass of aging products, nearly $95 \%$. The similarity of the compounds to those isolated from pulp was readily proven by identical retention times in thin layer chromatography (TLC), high-performance chromatography (HPLC), using toluene with $10 \%$ ethanol as the eluent in both cases, and by HR-MS. We were thus able to conclude that, first, model compound 1 was indeed a quite fitting model for the HexA unit in pulp and, second, it produced the same chromophoric compounds as the real-world pulp.

However, determination of the chemical structures of the compounds was far from being trivial. While mass spectrometry allowed deriving a chemical formula that corresponded with that derived from microanalysis (see experimental), fragmentation patterns were largely inconclusive. Evidently, all compounds contained no other elements than carbon, hydrogen, and oxygen. The molecular formulae derived indicated structures containing 10 (4), 12 (5), 15 (6), 18 (7), and $22(\mathbf{8})$ carbon atoms with rather low $\mathrm{C} / \mathrm{H}$ ratios of $2.5(\mathbf{4 , 6 , 7 )}, 3(\mathbf{5})$ and even $5.5(\mathbf{8})$.

\footnotetext{
${ }^{3}$ See the preceding part of this series for synthesis of the compound and its $13 \mathrm{C}$-isotopomers.
} 
This was clearly indicative of highly condensed structures which were in addition moderately oxygenated $(\mathrm{C} / \mathrm{O}$ ratios between 2 and 2.5). While not much information was expected from the ${ }^{1} \mathrm{H}$ NMR spectra because of the low $\mathrm{H}$ content, also most ${ }^{13} \mathrm{C}$ spectra NMR spectra were disappointingly void of indicative resonances, although well resolved. Except for 6, which showed the expected number of 15 carbons, all other compounds displayed peaks only for half the number of contained carbons. Evidently, those compounds were symmetric with a two-fold symmetry element and exclusively contained pairs of magnetically equivalent carbons. Moreover, all peaks were rather sharp and well-resolved so that inclusion compounds or valence isomerism were excluded. The opposite case was observed for compounds contained as key chromophores in pulps, such as 2,5-dihydroxy-1,4-benzoquinones (DHBQ) (Hosoya et al. 2013a) or 5,8-dihydroxy-1,4-naphthoquinone (Hosoya et al. 2013b), which are highly resonancestabilized moieties and hence give rather broad resonances in ${ }^{13} \mathrm{C}$ NMR. To conclude, we failed to derive the chemical structure of the five isolated compounds by a combination of the conventional analytical techniques (NMR, MS, IR, microanalysis).

Fortunately, all five compounds exhibited rather low solubilities in all kinds of organic solvents, especially polar ones, and a high tendency to form crystals suitable for crystal structure determination. For crystallization, the compounds were dissolved in hot glacial acetic acid and slowly cooled down to form large and well-shaped crystals. Recrystallization from both chloroform and toluene was also possible, but provided smaller specimens along with amorphous precipitates. The crystal structures of the five compounds are shown in Scheme 3, their chemical structures thus being established. The overlay with the drawn chemical formulae (Scheme 3, lower two rows) illustrates in a simple way an interesting aspect of the structure of the compounds: they are all completely planar. Almost all atoms lie perfectly well in the molecular plane, which renders the deviations to the drawn formula - of which the atoms also all lie in one plane, the paper plane-rather small. The torsion angles of $180{ }^{\circ} \mathrm{C}$ between the anellated rings confirm this simple illustration in a more "scientific" way. Such "two-dimensional" molecular arrangements are known, for instance, for graphene (Geim and Novoselov 2007), or polycylic aromatic hydrocarbons
(PAH) (Rieger and Muellen 2010) without substituents - even a substituent as small as a methyl group (or more precisely its hydrogens) would protrude from the molecular plane and add a "third dimension" to the molecule.

Properties, structure comparison, and reactivity of the chromophores

The degradation products derived from HexA are very potent chromophores. One molar solutions of all chromophores appear to be black; $10 \mu \mathrm{M}$ solutions are sufficiently dilute so that color differences can be seen. Even at this relatively low concentration, compounds $\mathbf{7}$ and $\mathbf{8}$ are still so strongly absorbing that their solutions appear nearly black. A $10 \mu \mathrm{M}$ equimolar mixture of the five compounds 4-8 appears black, while a $1 \mathrm{nM}$ (!) solution has a distinctly yellow overall color (see Fig. 1). This concentration translates into about $0.2 \mathrm{ppb}$ of each compound-this is roughly the realistic concentration range for commercial pulps. This simple experiment helps to illustrate why the identification of chromophores in pulps is such a tricky endeavor. The concentrations of the individual compounds are extremely low, but they are still very well perceptible to the human eye due to their high absorption coefficient, especially in the yellow range for which the human eye is particularly sensitive.

Compound 4 is a known compound-it has been reported as an intermediate in the synthesis of organic conductors (Takahashi and Kobayashi 2000). The other four compounds have not been described so far and represent novel structures. With the exception of 6, all compounds are symmetric with an inversion center $I$. This corresponds to the NMR data showing only "half the number" of carbon resonances for those four molecules, and to the mass spectra being poor in informative fragmentation mass peaks. The inversion center is evidently located in the geometric middle of the central 1,4-benzoquinone moiety in these molecules.

From the viewpoint of formal structure, compound 4 can be seen as a basic motif from which the other four compounds are derived: $\mathbf{5}$ has two carboxyl substituents in addition, 7 two anellated $p$-hydroxybenzoic acid moieties, and $\mathbf{8}$ two [2,3-f]-anellated benzofuran units. In all of these compounds the additional substituents (relative to 4) are introduced 
Scheme 3 Crystal structures of the HexAderived chromophore 4-8 and their overlay with the drawn formulae, illustrating the molecules to have perfectly planar, "twodimensional" geometries
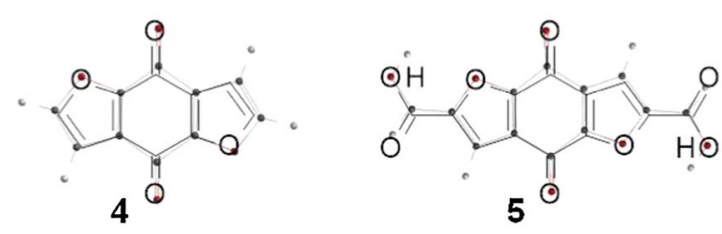

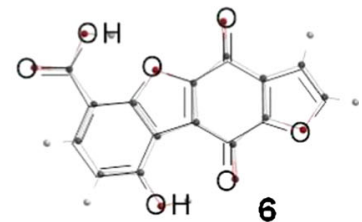

6
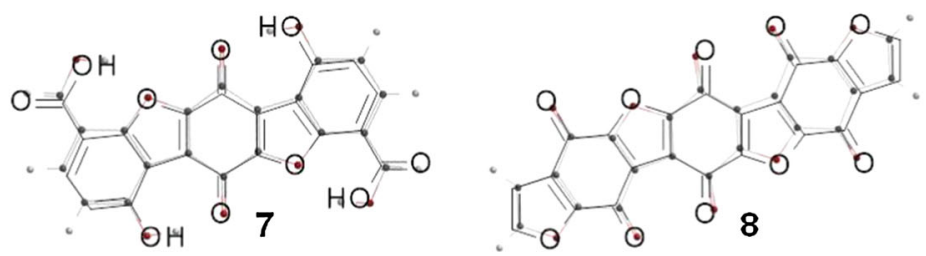
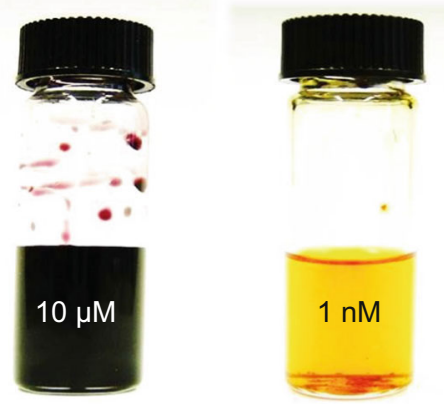

$1 \mathrm{nM}$

Fig. 1 Visible color of equimolar mixtures of the five compounds 4-8, nicely demonstrating their strongly chromophoric nature

in a symmetric way, so that the inversion symmetry is maintained. Only in compound 6 , just one $p$-hy-droxybenzoic acid substituent is appended onto $\mathbf{4}$, breaking the symmetry; $\mathbf{6}$ is the only non-symmetric compound among the isolated chromophores, with each of its 15 carbons giving an individual resonance in ${ }^{13} \mathrm{C}$ NMR since no magnetic equivalence due to symmetry reasons is prevailing.

The compounds are ladder-type oligomers of mixed furanoid/benzoid and mixed aromatic/quinoid nature. In all five chromophores, there are six-membered rings, either $p$-benzoquinone units or $p$-hydroxybenzoic acid units, anellated to (= fused with) furan units, i.e. the six-membered rings are amended with ethenyloxy units. By this anellation (lat. anellus = little ring), a $\mathrm{C}=\mathrm{C}$ double bond is at the same time part of the furan unit and the $p$-benzoquinone unit (or $p$ hydroxybenzoic acid, respectively). The furan-fused benzoquinones can also be conceived as furan moieties linked through carbonyl bridges; alternatively, they can be seen as partially oxidized phenolic units that are at the same time linked through orthopositioned aryl- $O$-aryl and aryl-aryl carbon bonds, or as 2,5-dihydroxy-1,4-benzoquinone units of which the hydroxyl groups are incorporated into fused heterocyclic aromatics (furan units) or etherified with phenol derivatives (2-hydroxy group of 2,4-dihydroxy-benzoic acid). It is interesting that every second furan unit is flipped by $180^{\circ}$ so that the two oxygens of the furans constitute at the same time two paraconfigured oxygen-substituents of the connecting sixmembered structures. The compounds seem to have a pronounced Janus character with regard to contained structural elements, and these ambiguities in the structural description already reflect the fact that the electronic structure will be rather complex and cannot be described in simple terms of (anti)aromaticity or substituent effects. The UV/Vis and fluorescence properties of the chromophores are currently studied, along with a computational treatment of their electronic structures. Preliminary results indicate interesting semiconducting properties of the parent compound and predict the derived (radical)-ions to be very stable or even persistent, both being interesting features for organic conductors. This indeed would be a completely new and unexpected facet of the unwanted "black sludge" from HexA degradation. This matter will be discussed in an upcoming account.

It is important to note that the structure of the compounds as identified does not agree with linear furan polymers as postulated in the literature (Gopalakrishnan et al. 2014; Gandini 2008, 2011; Gandini et al. 2008, 2009). Hitherto it was assumed that furfural and other furan derivatives link in a way that furan units are bridged by $\mathrm{C}_{1}$ links and form 
relatively flexible systems with extended conjugation that accounts for their color.

The structure determination of the chromophores might have yet another important implication. In biorefinery scenarios, hexoses and pentoses are often converted into furan derivatives that are to be further used as chemical building blocks. Formation of furfural from xylose/xylan and 5-hydroxymethylfurfural (HMF) from glucose/cellulose are the most wellknown examples. These conversions are always accompanied by formation of black condensation products that decrease the yield and cause problems in separation and purification. These dark by-products are usually denoted as "humins"-perhaps indicating similar color and complexity, but being structurally completely different from humic matter in soil. Such humins are formed by side reactions of mono-substituted or disubstituted furans, exactly as the HexAderived chromophores were. Without having done indepth structure determination of humins formed upon furfural or HMF production, we would like to point out the likelihood that those humins have very similar structures to the chromophores derived from HexA, thus being similar ladder-type oligomers of mixed aromatic and quinoid characters and mixed fivemembered and six-membered structural building blocks.

The formation mechanism of the chromophores from HexA seemed quite confusing at first glance. Since 2,5-dihydroxy-[1,4]-benzoquinone (DHBQ) was found to be a fundamental structural unit of chromophores in cellulosics (Hosoya et al. 2013a), it was logical to assume it to be a key element also in the HexA-derived chromophores, especially as HexA and DHBQ have the same number of carbon atoms. However, relative to DHBQ, compound $\mathbf{4}$ is distinguished by two additional ethenyl groups, and compound 5 by two additional propenoic acid motifs (see Scheme 4). The build-up of chromophores from such small fragments had been observed in the case of cellulose-derived chromophores, and thus seemed likely also for the HexA case. In 6 and 7, even one and two, respectively, whole $p$-hydroxybenzoic acid units are "added" onto 4 . The origin of such additional $\mathrm{C}_{2^{-}}, \mathrm{C}_{3^{-}}$or aromatic units remained puzzling for quite some time, especially the question why attachment of these small fragments to the central benzoquinone unit proceeded largely in a highly symmetric way. In the end, the formation mechanism could only be clarified
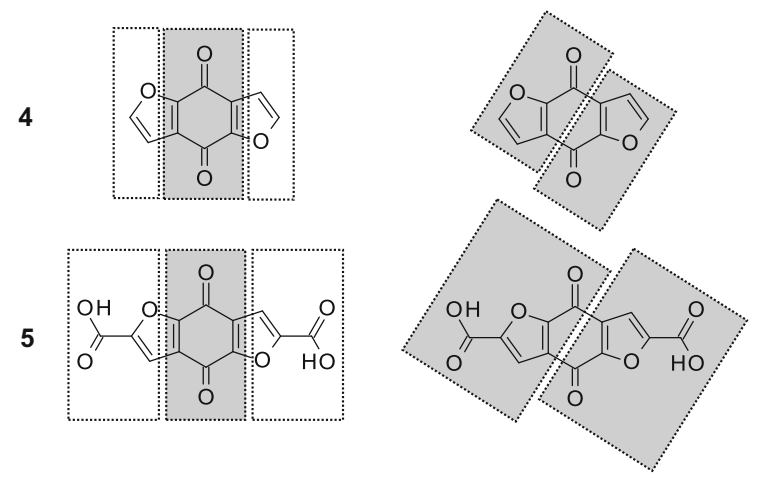

Scheme 4 Retrosynthetic attempts to illustrate the formation of HexA-derived chromophores 4 and 5. Left: "invalid" disconnection into synthons based on a central $p$-benzoquinone unit, right: valid deconstruction into 2-furanoyl and 2-furanoyl5-carboxylic acid units, respectively, as confirmed by isotopic labeling experiments (see Schemes 5,6)

by means of isotopically labeled HexA. For this purpose, six different ${ }^{13} \mathrm{C}$-isotopomers of HexA model compound $\mathbf{1}$ had been prepared (1a-1f) as described in the preceding part of this series.

The solution as to the formation mechanism was as simple as it was plausible. From a retrosynthetic point of view, the "trick" was to dissect the molecule not into $p$-benzoquinoid structures, but rather into furanoid fragments (Scheme 4). This way, compound $\mathbf{4}$ is composed of two 2-furanoyl (2-carbonylfuran) units that are linked in a symmetric way so that the carbonyl group of the first unit is linked to C-3 of the second, and the carbonyl group of the second linked to C-3 of the first furan. Compound $\mathbf{5}$ would be constructed in a similar way, with two 2-furanoyl-5-carboxylic acid units being symmetrically joined through carbonyl-C3 bonds. The two 2-furanoyl units making up $\mathbf{4}$ are two furan-2-carboxylic acid (3) units that are completely unchanged (apart from the two bonds linking them and a hydroxyl loss at the $\mathrm{COOH}$ group). The same applies to the 2-furanoyl-5-carboxylic acid units in 5: they represent almost unchanged 5-formylfuran-2-carboxylic acid (2) precursors.

How can now ${ }^{13} \mathrm{C}$-labeling help to support this formation mechanism of the chromophores? The ${ }^{13} \mathrm{C}$ isotopomers 1a-1f carry one carbon atom each with $99+\%{ }^{13} \mathrm{C}$ isotopic abundance. This atom remains like a marker throughout the chemical processes during chromophore formation. For instance, methyl $2-{ }^{13} \mathrm{C}$-4-deoxy- $\beta$-L-threo-hex-4-enopyranosid-uronic acid (1b) translates into the chromophore $\mathbf{4 b}$-or 


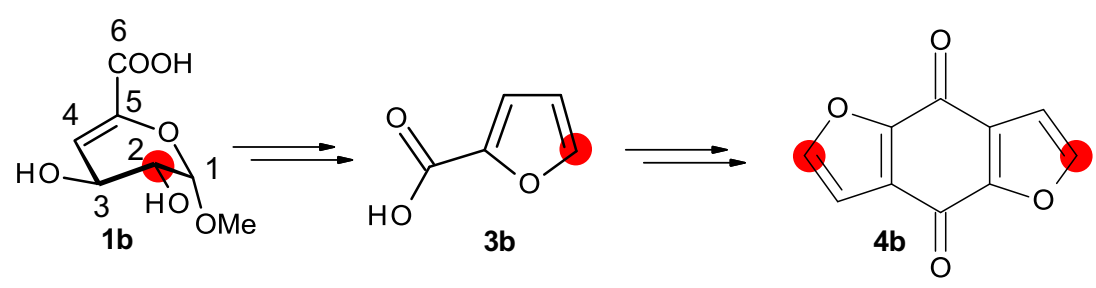

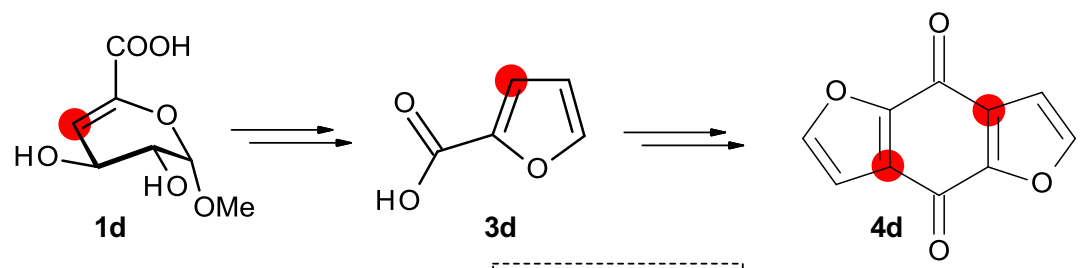

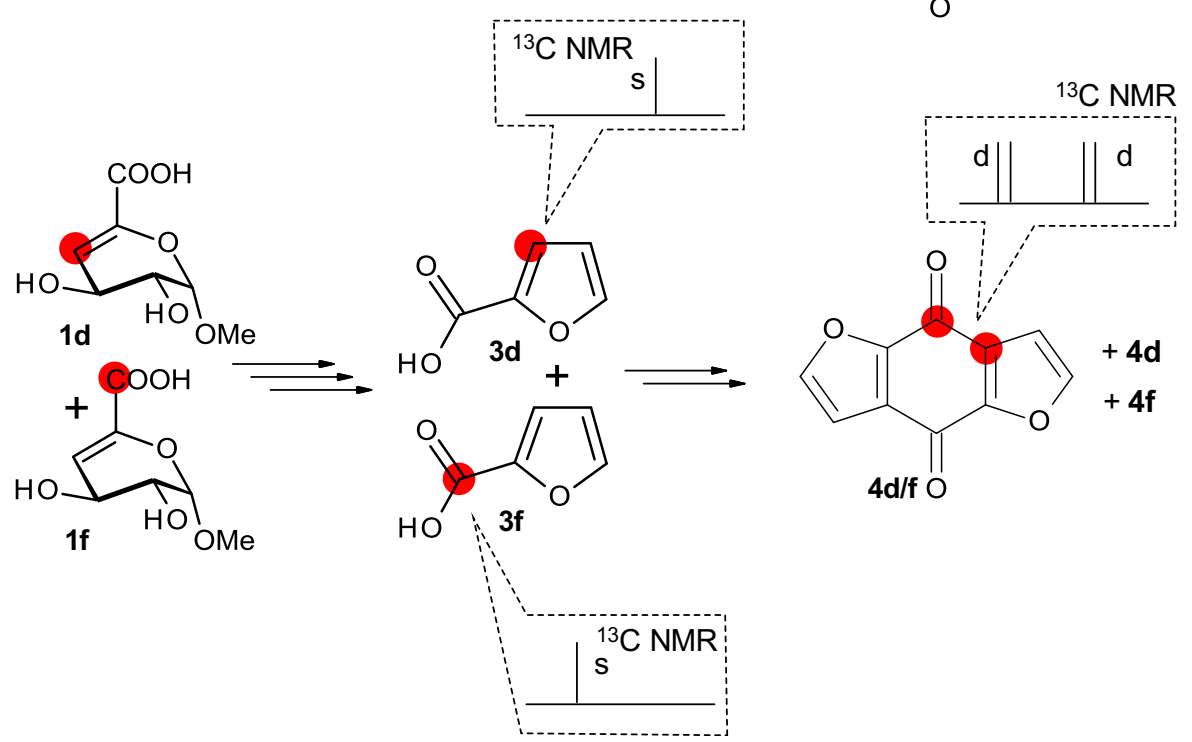

Scheme 5 Formation of isotopically labeled chromophores $4 \mathbf{b}$ and $\mathbf{4 d}$ from the respective ${ }^{13} \mathrm{C}$-labeled HexA compounds $\mathbf{1 b}$ and $\mathbf{1 d}$ via the intermediates $\mathbf{3 b}$ and $\mathbf{3 d}$. A mixture of two differently mono- ${ }^{13} \mathrm{C}$-labeled precursors, $\mathbf{1 d}+\mathbf{1 f}$, produces chromophore $4 \mathbf{d} / \mathbf{f}$, which shows a homonuclear ${ }^{1} J_{\mathrm{C}, \mathrm{C}}$ coupling,

methyl $\quad 4-{ }^{13} \mathrm{C}$-4-deoxy- $\beta$-L-threo-hex-4-enopyranosiduronic acid (1d) into $\mathbf{4 d}$ in an analogous way (Scheme 5). In each case, it was necessary, of course, to separate the respective chromophores $(\mathbf{4}, \mathbf{4 b}-\mathbf{4 f})$ from the other chromophores (5-8) that are formed alongside. The ${ }^{13} \mathrm{C}$ NMR spectra of the ${ }^{13} \mathrm{C}$-labeled products show one dominant resonance each-the carbon position carrying the label—while the other carbons are more or less "blanked out" by their roughly 100 -fold lower sensitivity. The fact that only one prominent resonance is seen proves that HexA was neatly converted into furanoid intermediates that combine in the presented way into the symmetrical thereby providing proof of these two carbons being directly connected, as well as of arrangement and linkage of the 2-furanoyl building blocks. The insets show a schematic representation of the ${ }^{13} \mathrm{C}$ NMR spectra of the precursors (two singlets) and the product (two doublets)

compound 4. This symmetry renders the two corresponding ${ }^{13} \mathrm{C}$-labels magnetically equivalent so that they produce only one peak.

A final proof of the linking and its positioning was provided by the following experiment: a mixture of two differently isotopically labeled HexA model compounds, namely methyl $5-{ }^{13} \mathrm{C}-4$-deoxy- $\beta$-Lthreo-hex-4-enopyranosiduronic acid (1e) and methyl $6-{ }^{13} \mathrm{C}-4$-deoxy- $\beta$-L-threo-hex-4-enopyranosiduronic acid (1f) was converted into the corresponding chromophores. In the starting mixture (1e/1f) as well as in the mixture of furanoid intermediates $(\mathbf{2} \mathbf{e} / \mathbf{2} \mathbf{f}$ and $\mathbf{3 e} / \mathbf{3 f})$ only singlets appear for the labeled position. In the 


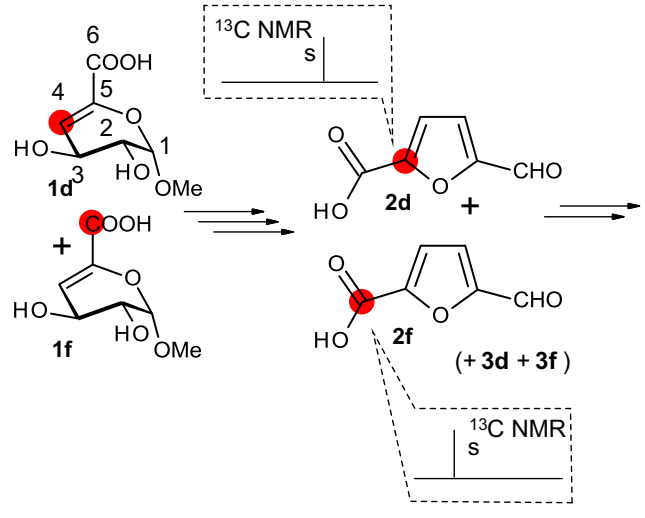

Scheme 6 A mixture of two differently mono- ${ }^{13} \mathrm{C}$-labeled precursors, $\mathbf{1 d}+\mathbf{1 f}$, produces chromophore $\mathbf{5 d} / \mathbf{f}$ via a mixture of the intermediates $\mathbf{2 d}$ and $\mathbf{2 f}$ and a dialdehyde intermediate that undergoes autoxidation. The product shows a homonuclear ${ }^{1} J_{\mathrm{C}, \mathrm{C}}$ coupling, thereby providing proof of the

chromophoric products, however, where two furanoyl building blocks combine, C-4 and C-5 (of the former HexA motif) form a $\mathrm{C}-\mathrm{C}$ bond. This is reflected by the occurrence of homonuclear $\mathrm{C}-\mathrm{C}$ coupling with ${ }^{1} J_{\mathrm{C}, \mathrm{C}}$ coupling constants of about $65 \mathrm{~Hz}$; thus the two labeled carbons do not appear as two singlets any longer, but as two doublets (see schematic drawing of the spectra in Scheme 5). In fact, the experiments, starting from a 1:1 mixture of $\mathbf{1 d}$ and $\mathbf{1 f}$, provided a mixture of the isotopomers $\mathbf{4 d}, \mathbf{4} \mathbf{f}$ and $\mathbf{4 d} / \mathbf{f}$ close to the theoretical ratio 1:1:2, which was similarly true for $\mathbf{5 d}$, 5f and 5d/f. In Schemes 5 and 6 the labeled carbon positions are indicated by red dots. These experiments confirmed the linkage patterns of the 2-furanoyl units in chromophores 4 (Scheme 5) and 5 (Scheme 6).

One interesting aspect of compound $\mathbf{5}$ was that the carboxyl groups of the former HexA units (C6) are engaged in the bonds making up the central $p$ benzoquinone unit (Scheme 6). The two "exo"carboxyl groups, in turn, actually represent the former C1-carbons in HexA, i.e. formyl (aldehyde) groups. However, in all isolated chromophores (and also in spectra of chromophore mixtures) we have never detected any aldehyde functions, which are readily discernible in NMR spectra by proton resonances $>9 \mathrm{ppm}$ and carbon resonances $>180 \mathrm{ppm}$. Evidently, the aldehyde groups have been oxidized to the corresponding carboxylic acids quite rapidly. An experiment in an inert atmosphere could verify that this oxidation is - at least in part-an autoxidation by

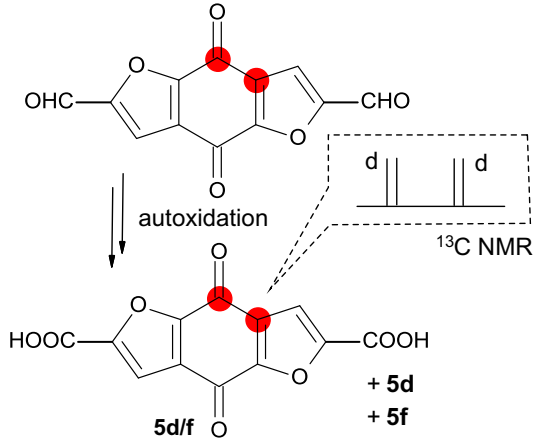

two labeled carbons being directly connected, and confirming arrangement and linkage of the 2-furanoyl-5-carboxylic acid building blocks. The insets show a schematic representation of the ${ }^{13} \mathrm{C}$ NMR spectra of the precursors (two singlets) and the product (two doublets)

oxygen: the chromophore mixtures initially contained aldehydes which had disappeared after $1 \mathrm{~h}$ in contact with air. But neither was it examined to which chromophores the unstable aldehydes belonged nor whether an additional redox process with an oxidizing intermediate was involved. Since the labeling experiments were able to unambiguously establish the position and linkages of the 2-furanoyl building blocks the minor issue of the oxidant in the C1-oxidation was not further followed.

In a similar way, the connections of building blocks were studied for chromophore 8 (see Scheme 7). HexA labeled in position 2 (methyl $2-{ }^{13} \mathrm{C}$-4-deoxy- $\beta$ L-threo-hex-4-enopyranosiduronic acid, 1b) produced two prominent singlets in the ${ }^{13} \mathrm{C}$ product spectrum coming from $2 \times 2$ magnetically equivalent carbons, while mixtures of $\mathbf{1 a} / \mathbf{1 d}, \mathbf{1 c} / \mathbf{1 f}$ and $\mathbf{1 d} / \mathbf{1 f}$ were used to confirm the three different inter-furanoyl linkages in the molecule (Scheme 7), again by the occurrence of two doublets in the NMR spectra caused by the homonuclear ${ }^{1} J_{\mathrm{C}, \mathrm{C}}$ coupling of the directly linked ${ }^{13} \mathrm{C}$ labeled carbons.

Analysis of the formation of chromophores $\mathbf{6}$ and $\mathbf{7}$ was somewhat more complicated: in these compounds, benzene rings ( $p$-hydroxybenzoic acid moieties) were present for which an analogous formation simply by condensation of furanoyl moieties could not be easily imagined. A similar approach as used for the linkage and formation analysis of the other chromophores-based on labeled HexA precursor and 

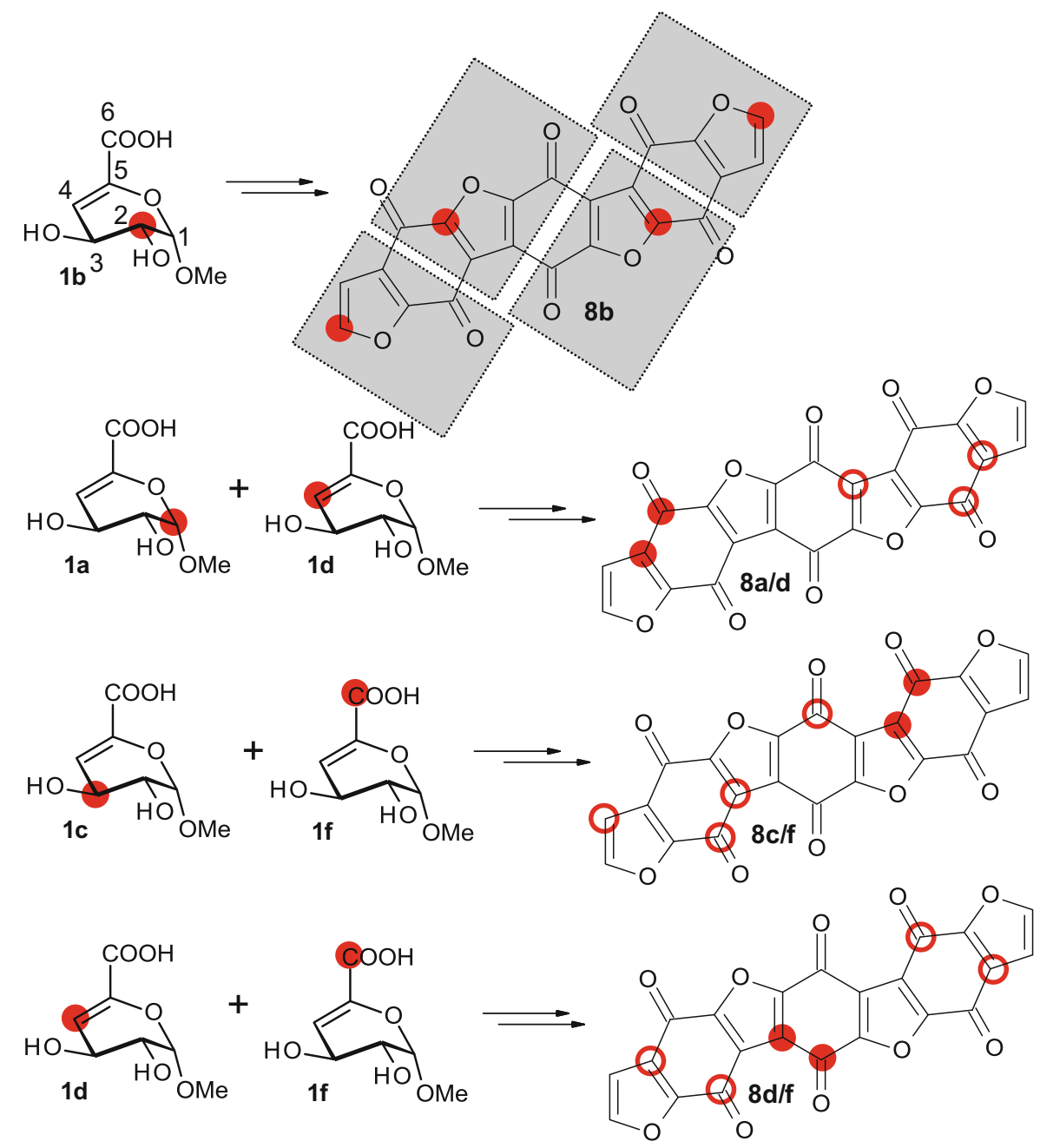

Scheme 7 Conversion of isotopically labeled HexA model $\mathbf{1 b}$ into the corresponding labeled chromophore $\mathbf{8 b}$, demonstrating this compound to be composed of four furanoyl units. Three pairs of differently mono- ${ }^{13} \mathrm{C}$-labeled precursors, $\mathbf{1 a}+\mathbf{1 d}$, $\mathbf{1 c}+\mathbf{1 f}$, and $\mathbf{1 d}+\mathbf{1 f}$, produce different isotopomers of chromophore $\mathbf{8}$ that contain four different ${ }^{13} \mathrm{C}$ labels each $(\mathbf{8 a} /$ $\mathbf{d}, \mathbf{8 c} / \mathbf{f}$ and $\mathbf{8 d} / \mathbf{f}$ ). While the precursors ${ }^{13} \mathrm{C}$ NMR spectra consist exclusively of singlets, the three differently labeled products show several different doublets because of the homonuclear ${ }^{1} J_{\mathrm{C}, \mathrm{C}}$ couplings of adjacent ${ }^{13} \mathrm{C}$ labels, thereby providing proof

position analysis of the ${ }^{13} \mathrm{C}$-labeles in the productsprovided the labels as shown in Scheme 8. Evidently, 6 consisted of an inner "core" of compound 4, to which one furanoyl $\left(\mathrm{C}_{5}\right)$ unit was appended to eventually give the anellated $p$-hydroxybenzoate unit in 6. Compound $\mathbf{7}$ was its binary counterpart, formed by anellation of two $p$-hydroxybenzoic acid units to the central compound $\mathbf{4}$ in a symmetric way. This way, 6 would be an intermediate in the formation of 7 , in of the three different types of inter-furanoyl linkages in compound 8. Red dots indicate the position of a ${ }^{13} \mathrm{C}$-label. Red circles indicate additional positions where only one of the two indicated carbons per furanoyl unit contains a label (with the one exception of compound $\mathbf{8 a} / \mathbf{d}$ : if the ${ }^{13} \mathrm{C}$-label at C-1 is lost as formic acid — see the preceding part of this paper series—one of the "outer" furanoyl units might contain no label at all so that in this case 8a/d contains only three labeled carbons). (Color scheme online)

which only one "side" of precursor 4 has reacted under $p$-hydroxybenzoate formation, while in 7 both "sides" had been involved in such an anellation. Compound $\mathbf{6}$ is thus made up of three furanoyl motifs, compound 7 of four. The linkage analysis for compound $\mathbf{6}$ is shown in Scheme 8, analysis for compound 7 was done analogously.

The assumption that $\mathbf{6}$ and $\mathbf{7}$ are derived from parent compound 4 offered at the same time a plausible 


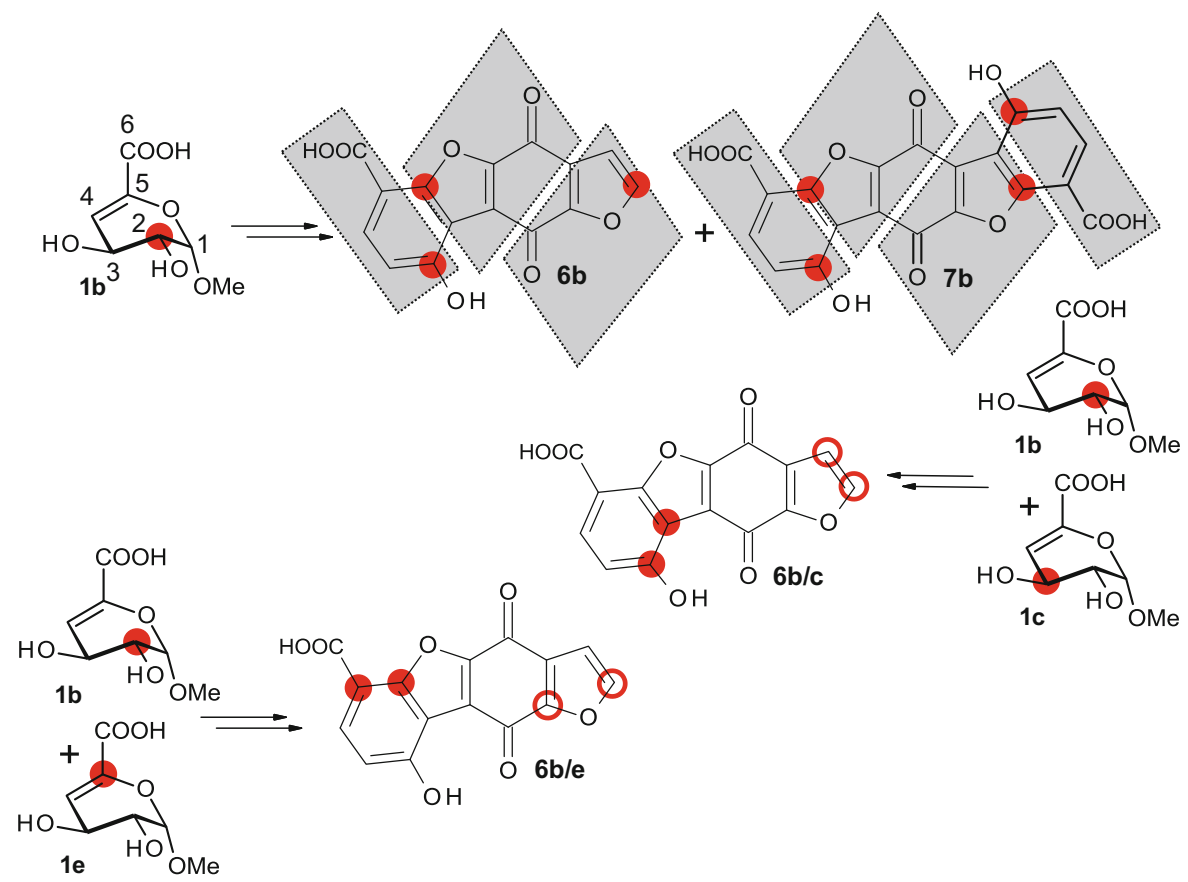

Scheme 8 Conversion of isotopically labeled HexA model $\mathbf{1 b}$ into the corresponding labeled chromophores $\mathbf{6 b}$ and $\mathbf{7 b}$, demonstrating these compounds to be composed of three and four furanoyl units, respectively. Two pairs of differently mono- ${ }^{13} \mathrm{C}$-labeled precursors, $\mathbf{1 b}+\mathbf{1 e}$ and $\mathbf{1 b}+\mathbf{1 c}$, produce different isotopomers of chromophore $\mathbf{6}$ that contain three different ${ }^{13} \mathrm{C}$ labels $(\mathbf{6 b} / \mathbf{c}$ and $\mathbf{6 b} / \mathbf{e})$, the linkage analysis of 7 having been performed analogously. While the precursors, ${ }^{13} \mathrm{C}$ NMR spectra consist exclusively of singlets, the differently

assumption as to the mechanism. As furan units have limited aromaticity and can be regarded as dienes (McKillip and Sherman 1980; Hoydonckx et al. 2012; Takebayashi et al. 1983), they readily undergo DielsAlder reactions, both as dienes and as dienophiles. In the most simple case, a furan derivative reacts "with itself" by assuming the role of ene and diene at the same time. That such a process is possible at all demonstrates that the energetic disfavoring by the loss of aromaticity is of little importance and readily overcompensated by entropic favoring (Gribble and Gilchrist 1998; Scaiano et al. 1988; Isidor et al. 1973). For compound 6, we can assume a mechanism shown in Scheme 9, with compound 4 undergoing a DielsAlder-type process with 5-formyl-furan-2-carboxylic acid (2) reacting as the corresponding diene. Intermediate $\mathbf{I}_{\mathbf{D A}}$ can be isolated when starting from a 1:1 mixture of $\mathbf{2}$ and $\mathbf{4}$. The regioselectivity of the diene approaching the dienophile in the $[4+2]$-cycloaddition is determined by the hydrogen bonding between labeled products show several different doublets because of the homonuclear ${ }^{1} J_{\mathrm{C}, \mathrm{C}}$ couplings of adjacent ${ }^{13} \mathrm{C}$ labels, thereby providing proof of how the $p$-hydroxybenzoic acid moiety is linked and structurally related to the furanoid precursor moieties. Red dots indicate the position of a ${ }^{13} \mathrm{C}$ label. Red circles indicate additional positions where only one of the two indicated carbons per furanoyl unit contains a label. (Color scheme online)

the carboxyl group to the furan ring's oxygen. Note that the intermediate has no endo-/exo-differences since 4 is planar and both approachable faces are equivalent. To form compound 7 , such sequence would proceed on both "sides" of precursor 4 , not just at one side only as it happens in the formation of $\mathbf{6}$.

The oxygen-bridged intermediate $\left(\mathbf{I}_{\mathbf{D A}}\right.$ in Scheme 9) would open up to eventually give a $p$ hydroxybenzoic acid motif, as indeed observed in $\mathbf{6}$ and 7. Detailed mechanistic investigations-which will be communicated in a separate account-have shown 5-formylfuran-2-carboxylic acid (2) to be the actual co-reactant of core chromophore 4 and thus precursor of 6 and 7. Furan-2-carboxylic acid (3) reacts in a similar Diels-Alder mode with $\mathbf{4}$, but the final products have anellated benzoic acid (not $p$ hydroxybenzoic acid) units and are thus missing the hydroxyl groups of $\mathbf{6}$ and $\mathbf{7}$ (they can be imagined as deoxy-6 and bisdeoxy-7). From the Diels-Alder intermediate $\mathbf{I}_{\mathbf{D A}}$, opening of the oxygen bridge is 


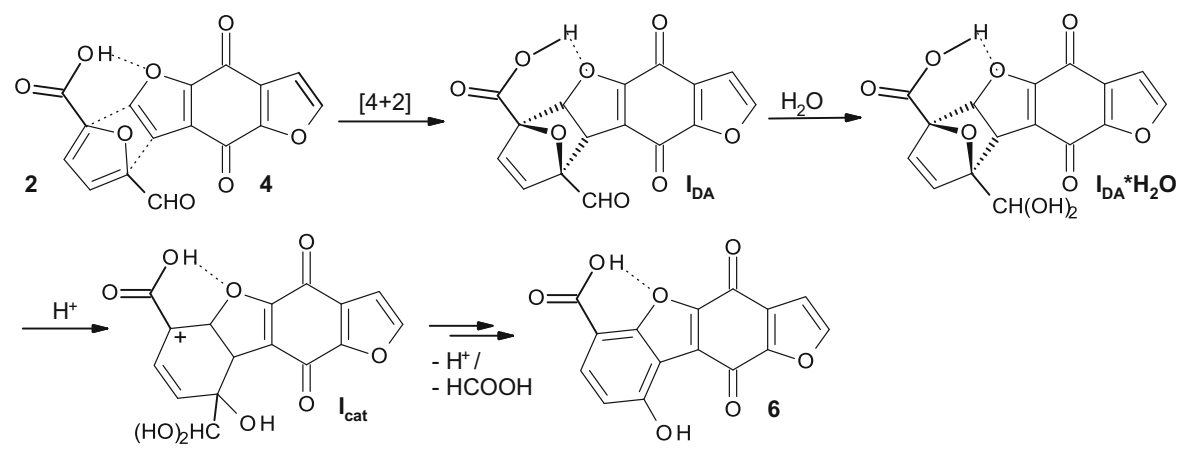

Scheme 9 Proposed mechanism of the formation of chromophore 6 from "core chromophore" 4, involving a DielsAlder reaction with diene 2 as the key step. The regioselectivity of this process is determined by the hydrogen bonding between

the first step that proceeds regioselectively, governed by the higher stability of the intermediate tertiary carbenium ion in $\alpha$-position to the carboxyl function, followed by loss of the neighboring proton. The formyl group is lost in an unconventional process similar to the deformylation described in the preceding paper. Why the formyl substituent is eliminatedwhich would be expected to be reasonably stableand not the hydroxyl group-which on the other hand would be reckoned to be rather elimination-pronewill be discussed in the above-mentioned treatise, also in connection with computational results. The final step is an oxidation of the cyclohexenone (hydroxycyclohexadiene) product driven by rearomatization, to establish the $p$-hydroxybenzoic acid moieties found in chromophores 6 and 7.

With the linkage analysis by means of isotopic labeling, all chromophores 4-8 were shown to consist of non-fragmented and largely unchanged furanoyl units (Schemes 5, 6, 7, 8, 9). It should be noted that this is in stark contrast to the chromophores from (over-)oxidized celluloses, such as 2,5-dihydroxy-1,4benzoquinone or 5,6-dihydroxy-1,4-naphthoquinone, which are formed by complex degradation of oxidized units in cellulose to $\mathrm{C}_{2}-\mathrm{C}_{4}$ units and recondensation of such small fragments. In the HexA-derived chromophores, the furanoyl degradation intermediates 2-furancarboxylic acid (3) and 5-formylfuran-2-carboxylic acid (2) are linked together and undergo follow-up transformations, but they remain largely intact without further degradation. They build up the chromophores like whole bricks a wall.

One particular feature of the chemistry of all five chromophores is their ease of reduction, not only to the the carboxyl group of the diene and the furan oxygen. The formation of $\mathbf{7}$ proceeds analogously, with both "sides" of compound 4 reacting in a Diels-Alder/rearomatization sequence

respective hydroquinones - this would be quite expectable for [1,4]-benzoquinones - but even further to the corresponding hydrocarbons, i.e. under loss of the oxygen functions, the resulting structures being shown in Scheme 10. The classical Clemmensen reduction (Clemmensen 1913), a method to reduce aldehydes and ketones to the corresponding alkanes in acidic media that usually uses (amalgamated) zinc and hydrochloric acid gives excellent yields above $90 \%$ in quite short reaction times. Conventionally, it gives good yields for dialkyl ketones and alkylaryl ketones, but it is rather problematic for diaryl ketones. The chromophores evidently represent a special case here where yields are unusually high. Interestingly, also reduction under alkaline conditions, for instance the standard Wolff-Kishner reduction (Szmant 1968; Wolff 1912), carried out with solid $\mathrm{KOH}$ and hydrazine in diethylene glycol, proceeds neatly with similarly high yields. It is rather unusual that a ketone can be reduced well to the alkane under both acidic and alkaline conditions; normally a clear preference

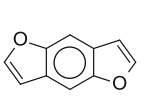

4R

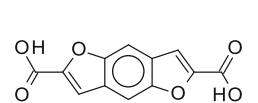

$5 R$

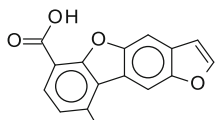

OH $6 R$
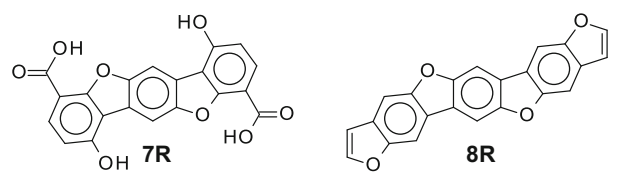

Scheme 10 Reduction of the HexA-derived chromophores providing the corresponding hydrocarbons $\mathbf{4 R}-\mathbf{8 R}$ remarkably easily and in excellent yields, both under acidic and alkaline reductive conditions 
for one type of medium is found. The reason for the particular ease and neatness of the reduction of the chromophores is evidently the high stability of reduction intermediates (both carbanions and carbenium ions) brought about by the extensive conjugation, and the extended aromaticity and exceptional stabilities of the resulting systems, which resemble those of polycyclic aromatic hydrocarbons (Mackay and Callcott 1998; Fetzer 2000; Harvey 1998). The redox behavior of the HexA-derived chromophores, along with the analytical data of the products $\mathbf{4 R - 8 R}$ (Scheme 10) will be discussed in detail in an upcoming account.

\section{Conclusion}

In the present description, the chemical structures of chromophores derived from hexeneuronic acids in cellulosic pulps (HexA), compounds 4-8, have been elucidated for the first time, and comprehensive analytical characterization was performed. There are no differences between the chromophores isolated from real-world pulp and those derived from HexA model compound $\mathbf{1}$, which clearly argues in favor of the suitability of this model compound. The number of chromophores generated from HexA is rather limited, with five chromophores accounting for more than $90 \%$ of the total mass of chromophores generated. In all isolated chromophores, largely intact 2-furanoyl units and 2-furanoyl-5-carboxylic acid units are contained. The small number and the intact building blocks are two distinct differences to chromophores isolated from cellulosic pulps as a consequence of oxidation and aging: in that case many different chromophores are formed by a far-reaching degradation of oxidized cellulose units and recondensation of fragments to the actual chromophores.

The HexA-derived chromophores 4-8 are laddertype, mixed furanoid/benzoid and mixed aromatic/ quinoid oligomers. They are strictly planar, highly conjugated compounds and highly chromophoric. Even a $1 \mathrm{nM}$ equilomar mixture of the five compounds still exhibits a yellow shade. The formation of the chromophores from the underlying 2-furanoyl units and 2-furanoyl-5-carboxylic acid units was clarified by means of ${ }^{13} \mathrm{C}$-isotopomers of model compound $\mathbf{1}$. By ${ }^{13} \mathrm{C}$ NMR units where two ${ }^{13} \mathrm{C}$ labels have become directly covalently linked in the product are easily identified by the homonuclear ${ }^{1} J_{\mathrm{C}, \mathrm{C}}$ coupling causing the appearances of doublets instead of only singlets that are found otherwise (without direct carboncarbon coupling between the labels). Similar to a puzzle where the arrangement of the individual pieces must fit to give the final picture, the number of furanoyl units per chromophore molecule and their linkage sites can be determined by this methodology, which was done for all five chromophores. The combination of isotopic labeling and NMR spectroscopy, as also used previously (Adelwöhrer et al. 2009; Krainz et al. 2010; Malz et al. 2007), once more proved to be a powerful tool in chromophore research.

In the follow-up papers in this series, we will explore the chemical behavior of the HexA-derived chromophores under bleaching conditions and their interrelation with the three key chromophore classes (Rosenau et al. 2005) found in cellulosic materials.

Acknowledgments Open access funding provided by University of Natural Resources and Life Sciences Vienna (BOKU). The authors would like to thank the Austrian Forschungsförderungsgesellschaft (FFG) for financial support through Project 847169 ("Chromophores II") and through the project FLIPPR ${ }^{2}$ (Future Lignin and Pulp Processing Research), along with its partner companies. We are indebted to the partner companies in this project for providing cellulosic pulps, valuable inputs to every-day pulping and bleaching practice as well as interesting discussions.

Open Access This article is distributed under the terms of the Creative Commons Attribution 4.0 International License (http:// creativecommons.org/licenses/by/4.0/), which permits unrestricted use, distribution, and reproduction in any medium, provided you give appropriate credit to the original author(s) and the source, provide a link to the Creative Commons license, and indicate if changes were made.

\section{References}

Adelwöhrer C, Takano T, Nakatsubo F, Rosenau T (2009) Synthesis of ${ }^{13} \mathrm{C}$-perlabeled cellulose with $>99 \%$ isotopic enrichment by a cationic ring-opening polymerization approach. Biomacromolecules 10:2013-2018

Bohrn R, Potthast A, Rosenau T, Sixta H, Kosma P (2005) Synthesis and testing of a novel fluorescence label for carboxyls in carbohydrates and cellulosics. Synlett 20:3087-3090

Bohrn R, Potthast A, Schiehser S, Rosenau T, Sixta H, Kosma P (2006) The FDAM method: determination of carboxyl profiles in cellulosic materials by combining group-selective fluorescence labeling with GPC. Biomacromolecules 7(6): 1743-1750

Chakar FS, Allison L, Ragauskas AJ, McDonough TJ (2000) Influence of hexenuronic acids on US bleaching operations. Tappi J 83:62 
Clavijo L, Cabrera MN, Kuitunen S, Liukko S, Rauhala T, Vuorinen T (2012) Changes in a eucalyptus kraft pulp during a mild acid treatment at high temperature. Papel 73:59-64

Clayton DW (1963) The alkaline degradation of some hardwood 4-O-methyl-D-glucuronoxylans. Svens Papperstidn 66:115-124

Clemmensen E (1913) Reduktion von Ketonen und Aldehyden $\mathrm{zu}$ den entsprechenden Kohlenwasserstoffen unter Anwendung von amalgamiertem Zink und Salzsäure. Ber Dtsch Chem Ges 46(2):1837-1843

Fetzer JC (2000) The chemistry and analysis of the large polycyclic aromatic hydrocarbons. Polycyclic aromatic compounds. Wiley, New York, 7(2). p 143. ISBN 0-47136354-5

Forsskåhl I, Tylli H, Olkkonen C (2000) Participation of carbohydrate-derived chromophores in the yellowing of highyield and TCF pulps. J Pulp Pap Sci 26(7):245-249

Gandini A (2008) Polymers from renewable resources: a challenge for the future of macromolecular materials. Macromolecules 41(24):9491-9504

Gandini A (2011) Furan monomers and their polymers: synthesis, properties and applications. Wiley, New York, pp 179-209

Gandini A, Silvestre AJD, Pascoal Neto C, Sousa AF, Gomes M (2008) The furan counterpart of poly(ethylene terephthalate): an alternative material based on renewable resources. J Polym Sci A Polym Chem 47(1):295-298

Gandini A, Coelho D, Gomes M, Reis B, Silvestre A (2009) Materials from renewable resources based on furan monomers and furan chemistry: work in progress. J Mater Chem 19(45):8656-8664

Geim AK, Novoselov KS (2007) The rise of graphene. Nat Mater 6(3):183-191

Gopalakrishnan P, Narayan-Sarathy S, Ghosh T, Mahajan K, Belgacem MN (2014) Synthesis and characterization of bio-based furanic polyesters. J Polym Res 21(1):1-9

Granstrom A, Eriksson T, Gellerstedt G, Roost C, Larsson P (2001) Variables affecting the thermal yellowing of TCFbleached birch kraft pulps. Nord Pulp Pap Res J 16(1):18-23

Gribble GW, Gilchrist TL (eds) (1998). A critical review of the 1997 literature preceded by two chapters on current heterocyclic topics. Progress in heterocyclic chemistry, vol 10, 1st edn. Pergamon Press, pp 1-360

Harvey RG (1998) Environmental chemistry of PAHs. In: Neilson A (ed) PAHs and related compounds: chemistry. The handbook of environmental chemistry. Springer, Berlin, pp 1-54. ISBN 978-3-540-49697-7

Hosoya T, French AD, Rosenau T (2013a) Chemistry of 2,5dihydroxy-[1,4]-benzoquinone, a key chromophore in aged cellulosics. Mini Rev Org Chem 10(3):309-315

Hosoya T, French AD, Rosenau T (2013b) Chemistry of 5,8dihydroxy-[1,4]-naphthoquinone, a key chromophore in aged cellulosics. Mini Rev Org Chem 10(3):302-308

Hoydonckx HE, Van Rhijn WM, Van Rhijn W, de Vos DE, Jacobs PA (2012) Furfural and derivatives. In:Ullmann's encycloped industrial chemistry. Wiley-VCH Verlag $\mathrm{GmbH} \& \mathrm{Co}$

Isidor JL, Brookhart MS, McKee RL (1973) Novel furan dimer. J Org Chem 38(3):612-613
ISO 2470-1 (2009) Paper, board and pulps-measurement of diffuse blue reflectance factor-part 1: indoor daylight conditions (ISO brightness)

Johansson MH, Samuelson O (1977) Epimerization and degradation of 2-O-(4-O-methyl- $\alpha$-D-glucopyranosyluronic acid)-D-xylitol in alkaline medium. Carbohydr Res 54:295-299

Korntner P, Hosoya T, Dietz T, Eibinger K, Reiter H, Spitzbart M, Roeder T, Borgards A, Kreiner W, Mahler AK, Winter H, Groiss Y, French AD, Henniges U, Potthast A, Rosenau $\mathrm{T}$ (2015) Chromophores in lignin-free cellulosic materials belong to three compound classes. Chromophores in cellulosics, XII. Cellulose 22(2):1053-1062

Krainz K, Hofinger A, Dietz T, Suess HU, Potthast A, Rosenau T (2010) Synthesis of methyl 4-O-methyl- $\beta$-D-ribo-hex-3ulopyranoside-1-13C and methyl 4-O-methyl- $\beta$-D-ribohex-3-ulopyranoside-3-13C as fragment analogues of oxidized cellulose units. Lett Org Chem 7:186-190

Li J, Gellerstedt G (1997) The contribution to kappa number from hexeneuronic acid groups in pulp xylan. Carbohydr Res 302(3-4):213-218

Mackay D, Callcott D (1998) Partitioning and physical chemical properties of PAHs. In: Neilson A (ed) PAHs and related compounds. The handbook of environmental chemistry. Springer, Berlin, pp 325-345. ISBN 978-3-642-08286-3

Malz F, Jäger C, Yoneda Y, Kosma P, Rosenau T (2007) Synthesis of methyl 4'-O-methyl- $\beta$-D-cellobioside- ${ }^{13} \mathrm{C}_{12}$ from D-glucose- ${ }^{13} \mathrm{C}_{6}$. Part 2: solid state NMR studies. Carbohydr Res 342:65-70

McKillip WJ, Sherman E (1980) Furan derivatives. Wiley, New York, pp 499-527

Potthast A, Röhrling J, Rosenau T, Borgards A, Sixta H, Kosma $\mathrm{P}$ (2003) A novel method for the determination of carbonyl groups in cellulosics by fluorescence labeling. 3. Monitoring oxidative processes. Biomacromolecules 4(3): 743-749

Potthast A, Rosenau T, Kosma P, Saariaho A-M, Vuorinen T (2005) On the nature of carbonyl groups in cellulosic pulps. Cellulose 12(1):43-50

Rieger R, Muellen K (2010) Forever young: polycyclic aromatic hydrocarbons as model cases for structural and optical studies. J Phys Org Chem 23(4):315-325

Röhrling J, Potthast A, Rosenau T, Lange T, Borgards A, Sixta H, Kosma P (2002a) A novel method for the determination of carbonyl groups in cellulosics by fluorescence labeling. 2. Validation and applications. Biomacromolecules 3(5): 969-975

Röhrling J, Potthast A, Rosenau T, Lange T, Ebner G, Sixta H, Kosma P (2002b) A novel method for the determination of carbonyl groups in cellulosics by fluorescence labeling. 1 . Method development. Biomacromolecules 3(5):959-968

Rosenau T, Potthast A, Milacher W, Hofinger A, Kosma P (2004) Isolation and identification of residual chromophores in cellulosic materials. Polymer 45(19): 6437-6443

Rosenau T, Potthast A, Milacher W, Adorjan I, Hofinger A, Kosma P (2005) Discoloration of cellulose solutions in $\mathrm{N}$ methylmorpholine- $N$-oxide (Lyocell). Part 2: isolation and identification of chromophores. Cellulose 12(2):197-208

Rosenau T, Potthast A, Kosma P, Suess HU, Nimmerfroh N (2007) Isolation and identification of residual 
chromophores from aged bleached pulp samples. Holzforschung 61(6):656-661

Rosenau T, Potthast A, Zwirchmayr NS, Hosoya T, Hettegger H, Bacher M, Krainz K, Yoneda Y, Dietz T (2017) Previous part of this series (Part XV of the series "Chromophores in cellulosic materials"): chromophores from hexeneuronic acids (HexA) - synthesis of model compounds and primary degradation intermediates. Cellulose (in press)

Scaiano JC, Wintgens V, Bedell A, Berson JA (1988) Absolute rates of dimerization and cycloaddition of 3,4dimethylenefuran and 3,4-dimethylenethiophene by nanosecond time-resolved spectroscopy. J Am Chem Soc 110(12):4050-4051

Sevastyanova O, Li J, Gellerstedt G (2006a) On the reaction mechanism of the thermal yellowing of bleached chemical pulps. Nord Pulp Pap Res J 21:188-192

Sevastyanova O, Li J, Gellerstedt G (2006b) Influence of various oxidizable structures on the brightness stability of fully bleached chemical pulps. Nord Pulp Pap Res J 21:49-53

Sjostrom E (2006) Do hexenuronic acid groups represent the majority of the carboxyl groups in kraft pulps? J Wood Chem Technol 26(3):283-288

Szmant HH (1968) Mechanism of the Wolff-Kishner reduction, elimination, and isomerization reactions. Angew Chem Int Ed Engl 7(2):120-128
Takahashi K, Kobayashi K (2000) Furan-fused TCNQ and DCNQI: synthesis and properties. J Org Chem 65:2577-2579

Takebayashi T, Iwasawa N, Mukaiyama T (1983) Synthetic control by internal interaction. The intramolecular DielsAlder reactions of furan derivatives and $\alpha, \beta$-unsaturated amides. Bull Chem Soc Jpn 56(4):1107-1112

TAPPI T 282 pm-07 (2009) Hexeneuronic acid content of chemical pulp

Teleman A, Harjunpaa V, Tenkanen M, Buchert J, Hausalo T, Drakenberg T, Vuorinen T (1995) Characterization of 4-deoxy- $\beta$-L-threo-hex-4-enopyranosyluronic acid attached to xylan in pine kraft pulp and pulping liquor by carbon-13 and proton NMR spectrometry. Carbohydr Res 272:55-71

Teleman A, Hausalo T, Tenkanen M, Vuorinen T (1996) Identification of the acidic degradation products of hexenuronic acid and characterization of hexenuronic acid-substituted xylooligosaccharides by NMR spectroscopy. Carbohydr Res 280:197-208

Wolff L (1912) Methode zum Ersatz des Sauerstoffatoms der Ketone und Aldehyde durch Wasserstoff. Liebigs Ann Chem 394:86-108 